\title{
ULTRASTRUCTURAL CHARACTERIZATION OF ATP SYNTHASE SUBUNIT B OVEREXPRESSION IN E. coli
}

\author{
Tracy L. Gales ${ }^{1}$, Marie J. Mazzulla ${ }^{2}$, Howard Kallender ${ }^{2}$, Fergal Hill ${ }^{3}$ and Beverly E. Maleeff ${ }^{1}$ \\ ${ }^{1}$ Safety Assessment, GlaxoSmithKline, King of Prussia, PA \\ ${ }^{2}$ Microbial Biochemistry, GlaxoSmithKline, Collegeville, PA \\ ${ }^{3}$ Avidis SA, Saint Beauzire, France
}

It is often difficult to obtain proteins from native sources in sufficient quantity for biophysical and structural studies. Recombinant techniques using transformed E. coli as a host have been the most successfully and widely used systems for overexpression of a range of proteins. Membrane proteins, however, present added difficulties in that overexpression often leads to death of the bacterium or formation of insoluble aggregates of non-native protein known as inclusion bodies ${ }^{1}$. In an attempt to overcome these difficulties two mutant hosts, C41(DE3) and C43(DE3), were developed from the parent E. coli strain BL21(DE3) with better tolerance for overexpression of membrane and toxic proteins $^{2}$. These mutant hosts were transformed using the pET-based plasmid pAVD 10 (Avidis SA, France), which contains the gene for $E$. coli membrane protein $\mathrm{F}_{1} \mathrm{~F}_{0}$ ATP synthase subunit $b$. Expression of this protein is under control of bacteriophage $\mathrm{T} 7$ promoter and $\mathrm{T} 7$ polymerase is controlled by isopropyl-2-D-thio-galactopyranoside (IPTG) inducible lac UV5 promoter. Proliferation of intracellular membranes (IM) in E. coli successfully overexpressing native membrane proteins has been reported previously ${ }^{3-5}$. This study assesses the ultrastructural manifestations of overexpression of ATP synthase subunit $b$ in BL21(DE3), C41(DE3), and C43(DE3) cell lines and correlates these with biophysical data.

pAVD 10 was introduced into BL21(DE3) cells (Novagen, Madison,WI) and the two mutant strains, $\mathrm{C} 41(\mathrm{DE} 3)$ and $\mathrm{C} 43$ (DE3) (Avidis) by electroporation. Cultures were grown at $37 \mathrm{C}$ to optical densities of 0.44-0.69 at $600 \mathrm{~nm}$. Aliquots of each were removed prior to induction (T0). Each culture was divided in half, IPTG added to $0.7 \mathrm{mM}$, and induction carried out at $37^{\circ} \mathrm{C}$ for $3 \mathrm{~h}$ or $25^{\circ} \mathrm{C}$ for $22 \mathrm{~h}$. Aliquots were taken for each timepoint (T37 and T25). For transmission electron microscopy (TEM), samples were fixed in $2.5 \%$ glutaraldehyde, $2 \%$ formaldehyde in $0.1 \mathrm{M}$ phosphate buffer, post-fixed with $1 \%$ osmium tetroxide, stained en bloc with $1 \%$ tannic acid, dehydrated in graduated ethanols and infiltrated and embedded in epoxy resin. Thin sections (approximately $85 \mathrm{~nm}$ ) of each sample were prepared and stained with uranyl acetate and lead citrate. The grids were examined with a JEOL 1200 EX TEM operating at $80 \mathrm{kV}$ and images collected using a Gatan 792 BioScan digital camera. For Coomassie gel analysis, $1.0 \mathrm{ml}$ cell pellets were suspended in 1x NuPAGE sample buffer containing reducing agent. Samples were boiled and $5 \mu \mathrm{l}$ of each sample were run on a $10 \%$ NuPAGE gel in MES running buffer.

Ultrastructural analysis revealed extensive IM in the transformed C41(DE3) and C43(DE3) strains, primarily at the T37 timepoint, but not in the BL21(DE3) strain (Figure 1). IM were located at one or both poles of a bacterium and presented as stacks of opposing tubules with an outer diameter of approximately $25 \mathrm{~nm}$. C41(DE3) and C43(DE3) cells exhibited similar IM networks. IM were seen infrequently in uninduced (T0) C41(DE3) and C43(DE3) cells. At the T25 timepoint most C41(DE3) and C43(DE3) cells exhibiting IM appeared dead. While BL21(DE3) cells contained no IM at any timepoints, areas of periplasmic degradation were seen in both induced samples. PAGE analysis of cell lysates showed heavy bands present at $\sim 17 \mathrm{kDa}$ at both induced time points for the C41(DE3) and C43(DE3) strains (Figure 2), which is in agreement with the known molecular weight of ATP synthase subunit $\mathrm{b}$ of $17.62 \mathrm{kDa}$. 
In this study TEM was a sensitive tool for detection of IM in E. coli overexpression systems. The presence of IM in the induced samples of C41(DE3) and C43(DE3) indicated protein overexpression and gel analysis suggested that the protein was predominately ATP synthase subunit b. Interestingly, the size and distribution of IM in this study closely matched the characteristics of IM of different membrane proteins overexpressed in $E$. coli in previous studies ${ }^{3,4}$. The pAVD 10/C43(DE3) expression system has also been shown to form IM when overexpressing ATP synthase subunit c, but only in tandem with overexpression of subunit $b^{5}$. It may be possible to overexpress other proteins in their native forms using this tandem method with ATP synthase subunit $b$.

\section{References}

[1] H Rogl et al., FEBS Letters 432 (1998) 21.

[2] B Miroux and JE Walker, J. Mol. Biol. 260 (1996) 289.

[3] JH Weiner et al., J. Bacteriology 158 (1984) 590.

[4] K von Meyenburg et al., EMBO J. 3 (1984) 1791.

[5] I Arechaga et al., FEBS Letters 482 (2000) 215.
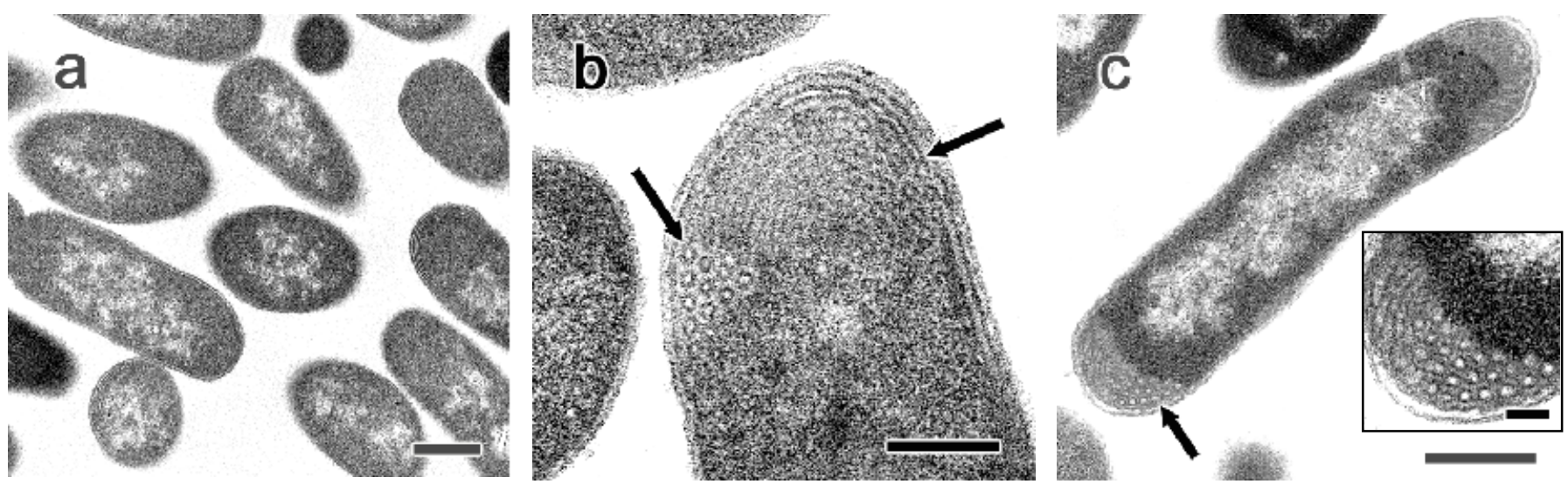

Figure 1. TEM micrographs of E. coli strains. C41(DE3) prior to induction (a), C41(DE3) at T37 (b), and C43(DE3) at T37 (c). Arrows indicate intracellular membranes. Bar (a and c) $=0.5 \mu \mathrm{m}$.

$\operatorname{Bar}(\mathrm{b})=200 \mathrm{~nm}$. Bar (c-inset) $=100 \mathrm{~nm}$.

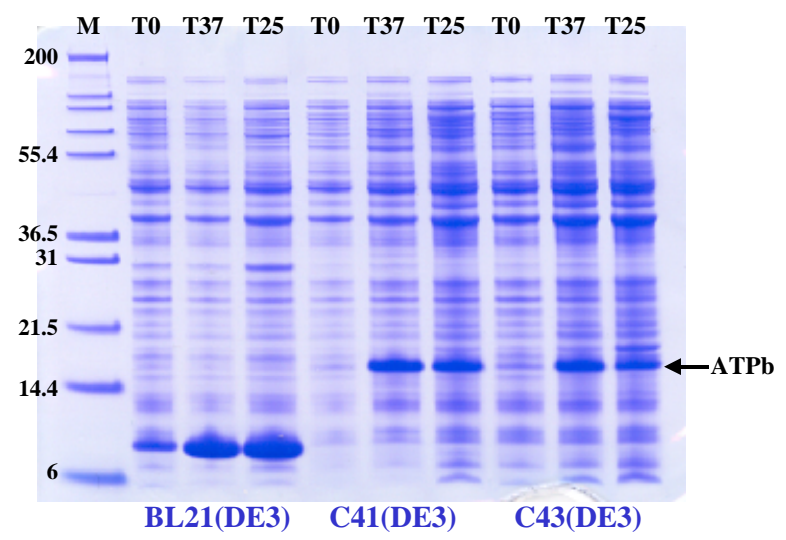

Figure 2. PAGE gel of pAVD10 expression in E. coli strains. Arrow indicates band for ATP synthase subunit b. Samples were run with molecular weight standards (M). 